\title{
Nuevas terapias en hemodiálisis: HFR aequilibrium
}

\author{
Premio Bellco a la investigación en terapias de hemofiltración
}

M Ma Dolores Álvarez Serrano, - Mª José Ludeña García,- Ma José Arnau Vives,

Unidad de Hemodiálisis. Hospital General Vall d’Hebron. Barcelona

\section{Resumen}

La técnica dialítica HFR Aequilibrium ${ }^{\circledast}$ es una forma de hemodiafiltración endógena que permite reinfundir al paciente el líquido ultrafiltrado. Se utiliza un dializador con doble cámara que permite separar el proceso convectivo del difusivo, más un cartucho de resina que capta básicamente la $\beta_{2}$ microglobulina y la angiogenina pero no así $\mathrm{Ca}++, \mathrm{Mg}++, \mathrm{HCO}$, aminoácidos esenciales, albúmina, glucosa, o fosfatos, lo que comporta la posibilidad de recuperar abundantes sustancias fisiológicas. Un software con un modelo matemático y un sensor gestionan automáticamente la extracción de sodio modificando la conductividad total durante toda la diálisis.

El objetivo de este trabajo es describir los resultados obtenidos al aplicar dicha técnica. Realizamos un estudio transversal descriptivo de enero a diciembre de 2008. Se determinaron valores al inicio y al final del estudio del perfil hemodinámico, hematológico, además de colesterol, PCR, albúmina, calcio y fósforo. Se tuvieron en cuenta incidencias, sintomatología e intervenciones registradas por enfermería.

Los resultados muestran un ajuste del sodio, potasio calcio y una tendencia a estabilizarse el fósforo. Destaca una importante disminución de triglicéridos frente a un ligero aumento de proteínas totales y albúmina, también disminuye la PCR. Por otra parte, a medida que avanza el tratamiento aumentan los

Correspondencia:

mjoarnau@hotmail.com valores de hemoglobina. La sintomatología intradialítica adversa disminuyó progresivamente.

Por tanto está técnica conlleva el aumento de prestaciones depurativas, con mejora de la tolerancia del tratamiento dialítico.

PALABRAS CLAVE:

- HEMODIAFILTRACIÓN

- HFR AEQUILIBRIUM ${ }^{\circledR}$

- REINFUSIÓN

- SINTOMATOLOGIA DIALITICA

\section{New therapies in haemodialysis: \\ HFR aequilibrium}

\section{Abstract}

The HFR Aequilibrium ${ }^{\circledR}$ dialysis technique is a form of endogenous haemodiafiltration that lets the patient be reinfused with the ultrafiltered liquid. A doublechamber dialyser is used which makes it possible to separate the convective process from the diffusive process, plus a resin cartridge that basically captures the $\beta_{2}$ microglobulin and the angiogenin but not $\mathrm{Ca}++, \mathrm{Mg}++, \mathrm{HCO}_{3}$, essential amino acids, albumin, glucose, or phosphates, which makes it possible to recover abundant physiological substances. Software with a mathematical model and a sensor handles the extraction of sodium automatically, modifying the total conductivity throughout the dialysis.

The aim of this study is to describe the results obtained on applying this technique. We carried 
out a descriptive transversal study from January to December

2008. Values were determined at the beginning and at the end of the study of the haemodynamic profile, haematological profile, as well as cholesterol, CRP, albumin, calcium and phosphorus. Incidents, symptomatology and interventions recorded by nursing staff were taken into account.

The results show an adjustment of sodium, potassium, calcium and a tendency to stabilize in phosphorus. There was an important reduction in triglycerides compared to a slight increase in total proteins and albumin, the CRP also fell. On the other hand, as the treatment advanced, the values of haemoglobin increased. Intradialytic symptomatology decreased progressively.

Accordingly, this technique involves an increase in purifying functions, with an improvement in the tolerance of the dialysis treatment.

\section{KEY WORDS:}
- HAEMODIAFILTRATION
- HFR AEQUILIBRIUM ${ }^{\circledR}$
- REINFUSION
- DIALYTIC SYMPTOMATOLOGY

primera parte del filtro está formada por una membrana de alta permeabilidad donde se realiza una ultrafiltración para obtener una depuración de tipo convectivo con la eliminación de sustancias de peso molecular medio/ alto. La segunda parte del filtro de baja permeabilidad, con paso de baño de diálisis, realiza una depuración por difusión de pequeñas moléculas. A éste sistema clásico de PFD se ha colocado en el circuito de ultrafiltración un cartucho de $40 \mathrm{ml}$ de resina en solución fisiológica con gran superficie adsorbente $\left(700 \mathrm{~m}^{2} / \mathrm{ml}\right.$ resina). Esto permite que el agua plasmática ultrafiltrada se reinfunda entre los dos filtros lo que supone un circuito de ultrafiltración cerrado donde el líquido de reinfusión que se utiliza es endógeno.

Permite por tanto la eliminación de "toxinas perjudiciales" por adsorción y la reinfusión de importantes moléculas fisiológicas.

\section{Objetivos}

Describir los resultados obtenidos al aplicar la técnica HFR-Aequilibrium en nuestro servicio, teniendo en cuenta:

- Observar y valorar los cambios analíticos producidos con la aplicación de la técnica.

- Valorar signos, síntomas e incidencias acontecidos durante las sesiones de HD.

\section{Material y método}

El material utilizado no difiere demasiado del utilizado en una hemodiálisis convencional, éste consta de un dializador de doble cámara "HFR-17" formado por un filtro de alta permeabilidad de Polyphenylene HF de 0,7 $\mathrm{m}^{2}$ de superficie y un segundo de baja permeabilidad de Polyphenylene HF de 1,7 $\mathrm{m}^{2}$ de superficie. Además se ha de disponer de un cartucho de resina y de un juego de líneas arterial y venosa, más una tercera línea que configura el circuito cerrado de ultrafiltración/reinfusión.

Funcionamiento de la técnica. Obtenemos mediante una luz sangre que se dirige a la cavidad superior del dializador (entrada de sangre), aquí mediante un proceso convectivo se produce una ultrafiltración de líquido, la sangre sigue su camino descendente, y éste es conducido al cartucho adsorbente donde se libera de toxinas, para ser de nuevo reinfundido en la parte 
media del dializador dando como resultado la reposición de la volemia del paciente. El líquido ultrafiltrado pasa por detectores de seguridad antes de ser reinfundido con objeto de evitar retrofiltraciones, fugas o aire. La sangre junto con el líquido endógeno reinfundido son sometidos a un proceso difusivo en la parte inferior del dializador como en una HD convencional (figura 1).

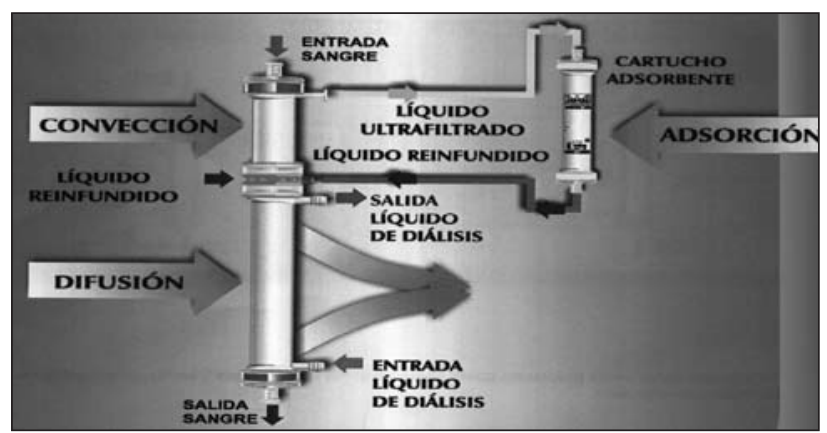

Figura 1. Esquema de la técnica

Este proceso permite que solutos de bajo peso molecular como creatinina y ácido úrico se absorban de manera más selectiva y que mediante el líquido de ultrafiltrado sean reinfundidos urea, bicarbonatos, fosfatos, iones y glucosa.

Realizamos un estudio transversal de carácter descriptivo con la finalidad de cumplir con los objetivos previamente marcados. El periodo de estudio comprendió los meses de junio 2007 y diciembre del 2008. La población de nuestro estudio la dividimos en dos grupos. El primero formado por pacientes con mala tolerancia en general a la hemodiálisis (grupol) y otro compuesto por individuos sin problemas relacionados con el tratamiento (grupo 2).

Se tomaron valores hemodinámico, hematológicos y bioquímicos, tales como hemoglobina, hematocrito, ferritina, colesterol, PCR, albúmina, calcio y fósforo, por los importantes efectos que puede comportar un aumento 0 descenso de los mismos.

Realizamos una recogida de datos analíticos previos a la inclusión de los pacientes en el programa (junio 2007 a enero 2008) y otra que se correspondía con el período de aplicación de la técnica (enero 2008 a diciembre 2008). Así como registros enfermeros que nos aportaron valores hemodinámicos durante las diferentes sesiones de HD, así como las incidencias e intervenciones habidas en el transcurso de las mismas.
Para el análisis estadístico sobre la base de datos descriptivos realizamos una distribución de las variables cualitativas y cuantitativas con el programa Microsoft Excel 2007.

\section{Resultados}

Del grupo 1, formado por pacientes con edad media de 65 años y mala tolerancia a la HD, obtuvimos respecto al perfil hemodinámico los resultados que se muestran en la figura 2. Como podemos observar la variación hemodinámica pre-inclusión o con aplicación técnica HFR-Aequilibrium no fue significativa, a excepción del periodo Inter-HD con tratamiento HFR en el cual la tendencia fue a subir ligeramente. Destacar que los pacientes se mantuvieron en todos los casos asintomáticos a pesar de haberse producido en alguna ocasión hipotensión arterial. Únicamente en casos esporádicos suspendimos como medida cautelar la UF, en algunos pacientes diabéticos con patología vascular, pudiendo conseguir el peso seco del paciente aún cuando éste sobrepasaba la tasa recomendada.

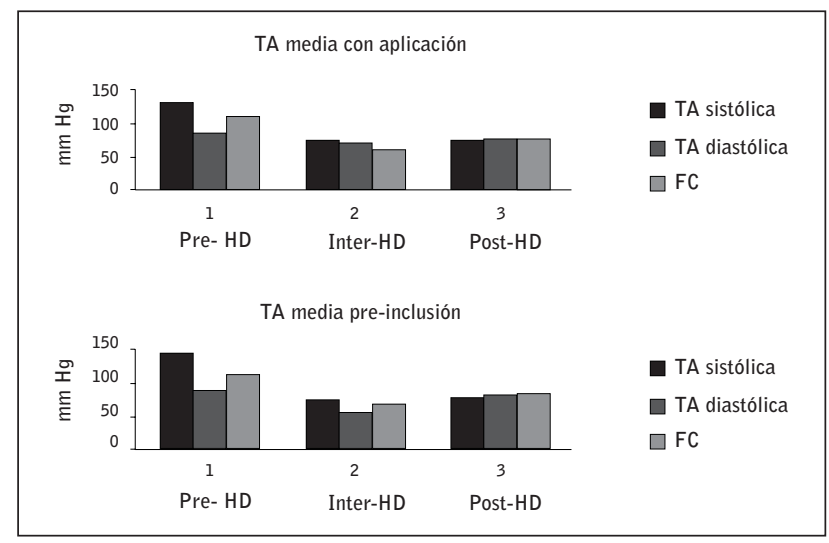

Figura 2. Valores deTA pre y post estudio (grupo 1)

Los signos y síntomas disminuyeron significativamente en el periodo intradialítico en los pacientes sometidos a tratamiento con mayor incidencia a medida que este avanzaba. En cuanto a los resultados analíticos destacamos:

Con los iones sodio y potasio observamos que existió una variación significativa con respecto a la pre-inclusión al tratamiento; a nivel de $\mathrm{K}^{++}$se produjo una estabilidad en los dos grupos con parámetros dentro de la normalidad $(3,68-4,86 \mathrm{mmol} / \mathrm{L})$ y en el caso del $\mathrm{Na}^{++}$conseguimos el nivel que nos propusimos de inicio, lo que representó que los pacientes tuvieran menos sensación de sed. 
Con respecto al calcio y fósforo, el $\mathrm{Ca}++(8,9-10,3 \mathrm{mg} /$ $\mathrm{dl}$ ), en el grupo de pacientes con mala tolerancia a la HD se estabilizó y mantuvo dentro del rango de normalidad. En cuanto al fósforo la tendencia fue hacia el equilibrio si bien no se consiguieron los parámetros deseados. Del estudio se desprendieron factores relacionados con la toma irregular de la medicación en los miembros del grupo 1 .

Con referencia al estado nutricional destacamos una importante disminución de triglicéridos (figura 3) frente a un ligero aumento de proteínas totales y albúmina Se observa una importante disminución de PCR lo que conlleva un menor riesgo de desarrollar enfermedad cardiovascular.

A igual dosis de eritropoyetina e incluso reduciendo ésta a medida que avanza el tratamiento conseguimos un aumento en los valores de hemoglobina (figura 4) y una tendencia a la regularidad en el acumulo de ferritina (figura 5).

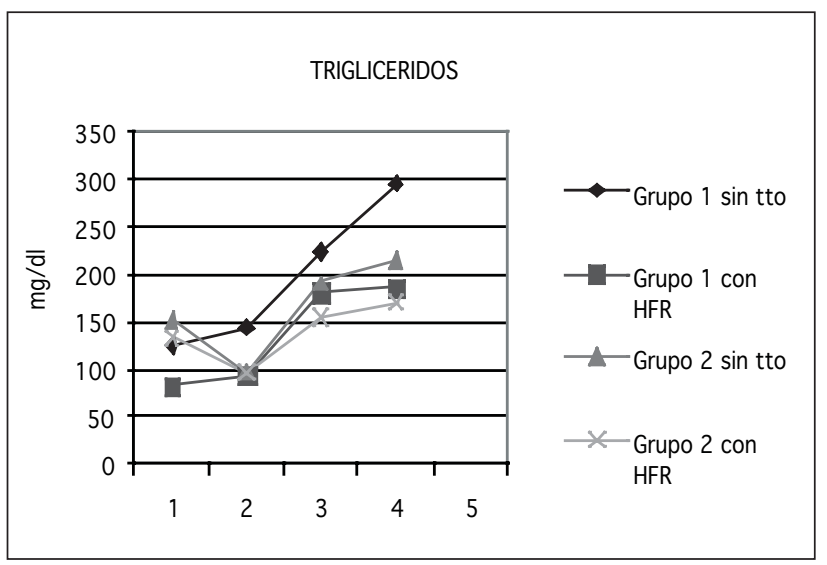

Figura 3. Valores de triglicéridos

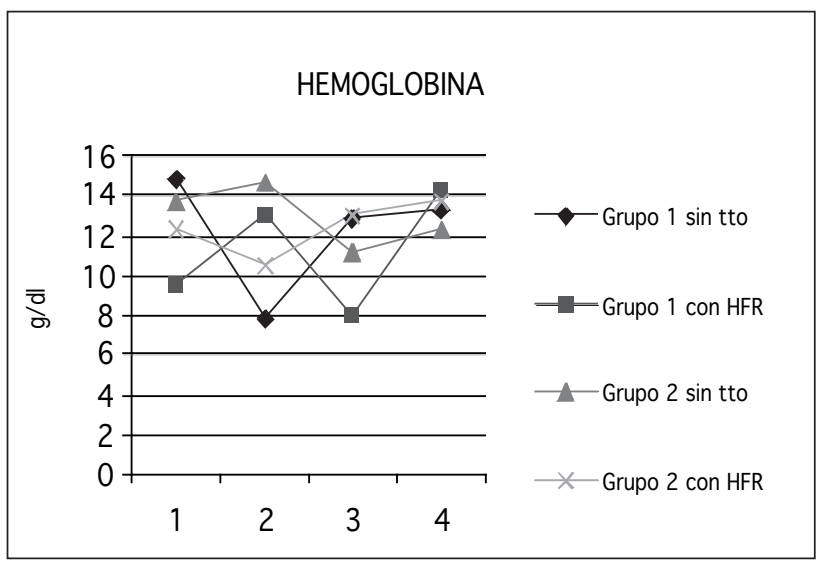

Figura 4. Valores de hemoglobina

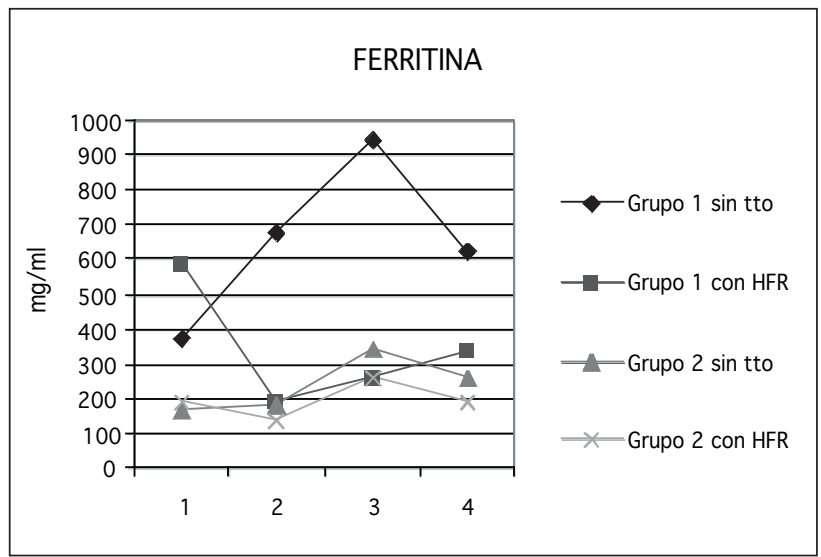

Figura 5. Valores de ferritina

\section{Discusión/conclusiones}

Existe una diferencia importante en los resultados obtenidos antes y después de someterse los pacientes al tratamiento. Se produce una mejoría en su estado general, incluso aquellos que mantenían una buena tolerancia a la HD, manifestaron sentirse más vitales desde que se sometían a la terapia.

Los resultados se reflejaron en su aspecto físico y en la disminución de la sintomatología que padecían durante las sesiones y fuera de éstas. Existen diferencias estadísticas entre los valores que obtuvimos antes y después de la aplicación de la terapia.

De aquellos en los que la tolerancia era mala destacar el caso de un paciente con diagnóstico de neuropatía diabética en el cual conseguimos durante la técnica disminuir el nivel de dolor. Hecho que tiene su lógica si tenemos en cuenta que el tratamiento mejora el estado nutricional, disminuye la inflamación crónica, la hipotensión y la hipovolemia del enfermo.

Para concluir decir que el uso de esta práctica conlleva el aumento de prestaciones depurativas, con mejora a la tolerancia del tratamiento dialítico lo que representa una HD más fisiológica para el paciente.

\section{Agradecimientos}

A todos los pacientes que con su colaboración han hecho posible este estudio, y a todos los compañeros de la Unidad de Hemodiálisis que han ayudado en este proyecto. 


\section{Bibliografía}

1. De Francisco ALM et al. Haemodiafiltración with sorbent-regenerated ultrafiltrate as replacement fluid: a multicenter study. European Renal Association-European. Dialysis and Transplant Association, 1997.

2. Ghezzi PM, Gervasio R. Hemodiafiltración Without Replacement Fluid. An Experimental Study. ASAIO Journal, 1992.

3. Hhezzi P.M. Emodiafiltrazione con reinfusione endogena. Gia Direttore delñ I'U.0. di Nefrologia e Dialisi, A.0. "Croce e Carle", Cuneo. Società Italiana di Nefrología.
4. Locatelli F, Stefoni S, Petitclerc T. Efectos de la terapia HFR Aequilibrium en la estabilidad cardiovascular: Estudio europeo multicéntrico.

5. Pacitti A, Bermond F. La reinfusione esogena vs quella endogena in emodiafiltrazione. S.C. di Nefrologia, Dialisi, Trapiento Rene Ospedale "Regina Marguerita". Torino.

6. Valderrábano F. Tratado de hemodiálisis. Editorial Médica Jinés S.L., 1999. 